\title{
ARTICLE
}

Molecular Diagnostics

\section{Pre-operative cellular dissociation grading in biopsies is highly predictive of post-operative tumour stage and patient outcome in head and neck squamous cell carcinoma}

\author{
Moritz Jesinghaus ${ }^{1,2}$, Katja Steiger ${ }^{1}$, Fabian Stögbauer ${ }^{1}$, Bernhard Haller ${ }^{3}$, Andreas Kolk ${ }^{4}$, Ulrich Straßen ${ }^{5}$, Anja Pickhard ${ }^{5}$, \\ Markus Wirth ${ }^{5}$, Miguel Silva ${ }^{2}$, Jan Budczies ${ }^{6}$, Aaron Becker von Rose ${ }^{7}$, Björn Konukiewitz ${ }^{1}$, Peer Kuhn ${ }^{1}$, Konrad Klinghammer ${ }^{8}$, \\ Hendrik Dapper ${ }^{9}$, Stefan Münch ${ }^{9}$, Stephanie E. Combs ${ }^{9,10,11}$, Wilko Weichert ${ }^{1,10}$ and Melanie Boxberg ${ }^{1}$
}

\begin{abstract}
BACKGROUND: Pre-operative treatment planning in head and neck squamous cell carcinoma (HNSCC) is mainly dictated by clinical staging, which has major shortcomings. Histologic grading is irrelevant due to its lack of prognostic impact. Recently, a novel grading termed Cellular Dissociation Grade (CDG) based on Tumour Budding and Cell Nest Size was shown to be highly prognostic for resected HNSCC. We aimed to probe the predictive and prognostic impact of CDG in the pre-operative biopsies of HNSCC. METHODS: We evaluated CDG in $n=160$ pre-therapeutic biopsies from patients who received standardised treatment following German guidelines, and correlated the results with pre- and post-therapeutic staging data and clinical outcome.

RESULTS: Pre-operative CDG was highly predictive of post-operative tumour stage, including the prediction of occult lymph node metastasis. Uni- and multivariate analysis revealed CDG to be an independent prognosticator of overall, disease-specific and disease-free survival $(p<0.001)$. Hazard ratio for disease-specific survival was 6.1 (11.1) for nG2 (nG3) compared with nG1 tumours. CONCLUSIONS: CDG is a strong outcome predictor in the pre-treatment scenario of HNSCC and identifies patients with nodalnegative disease. CDG is a purely histology-based prognosticator in the pre-therapeutic setting that supplements clinical staging and may aide therapeutic stratification of HNSCC patients.
\end{abstract}

British Journal of Cancer (2020) 122:835-846; https://doi.org/10.1038/s41416-019-0719-8

\section{BACKGROUND}

Head and neck squamous cell carcinoma (HNSCC) is one of the most common cancers worldwide with $\sim 500.000$ new cases annually. ${ }^{1}$ HNSCC arise in different anatomical areas of the head and neck region. Human Papilloma Virus (HPV) - negative cases are particularly located within the oral cavity, hypopharynx and larynx. ${ }^{2,3}$ Overall, the prognosis of HNSCC, especially of HPV-negative cases, is unfavourable with a 5-year survival of patients on average below $50 \% .^{4,5}$ To date, tumour stage according to clinical and pathological TNM and UICC classification provides the most important prognostic information for patient outcome. ${ }^{3-5}$ However, pre-operative clinical assessment of tumour size (cT stage) and nodal involvement (cN stage) is discordant from pathological pT- and $\mathrm{pN}$ stage in nearly half of cases. ${ }^{6}$ Estimates assume that in early-stage oral squamous cell carcinoma (SCC) without clinically or radiologically apparent nodal metastasis $20-40 \%$ of patients contain occult metastasis at the time of diagnosis. $^{7-9}$ As accurate routine biomarkers besides clinical staging for detection of such occult metastasis are still lacking, it is a matter of an ongoing debate which patients could be treated adequately and sufficiently without neck dissection in order to avoid overtreatment and in which patients to perform neck dissection in order to detect clinically non-apparent nodal metastasis and therewith improve prognosis. ${ }^{10-12}$

Currently, histopathologic grading does not play a major role in clinical decision making as current histopathological grading schemes according to the World Health Organization (WHO) possess major shortcomings regarding prognostic patient stratification and reproducibility. ${ }^{13-17}$ In an effort to resolve this issue, our group recently proposed a new histopathological grading scheme termed Cellular Dissociation Grade (CDG) for oral, hypopharyngeal and laryngeal SCC, which is based on the parameters Tumour Budding and Cell Nest Size. These parameters describe the extent of Cellular Dissociation of a given cancer, both from a quantitative (Tumour Budding) and qualitative (Cell Nest Size) angle. CDG has not only been shown to be highly prognostic

\footnotetext{
${ }^{1}$ Institute of Pathology, Technical University Munich, Munich, Germany; ${ }^{2}$ German Cancer Consortium (DKTK), Partner Site Munich, Institute for Translational Cancer Research, Munich, Germany; ${ }^{3}$ Institute for Epidemiology and Statistics, Technical University Munich, Munich, Germany; ${ }^{4}$ Department of Head and Neck Surgery, Klinikum Rechts der Isar, Munich, Germany; ${ }^{5}$ Department of Otolaryngology, Klinikum Rechts der Isar, Munich, Germany; ${ }^{6}$ Institute of Pathology, University of Heidelberg, Heidelberg, Germany; ${ }^{7}$ III Medizinische Klinik, Klinikum rechts der Isar, Technical University Munich, Munich, Germany; ${ }^{8}$ Department for Hematology and Oncology, Charite University Medicine, Berlin,

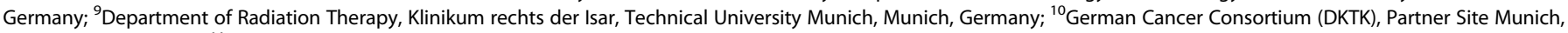
Munich, Germany and ${ }^{11}$ Institute of Innovative Radiotherapy (iRT), Helmholtz Center Munich, Munich, Germany

Correspondence: Melanie Boxberg (Melanie.Boxberg@tum.de)

These authors contributed equally: Wilko Weichert, Melanie Boxberg
}

Received: 20 October 2019 Revised: 3 December 2019 Accepted: 18 December 2019

Published online: 15 January 2020 
Table 1. Distribution and survival associations for clinicopathological data, histomorphological parameters and Cellular Dissociation Grade in biopsies and resection specimens.

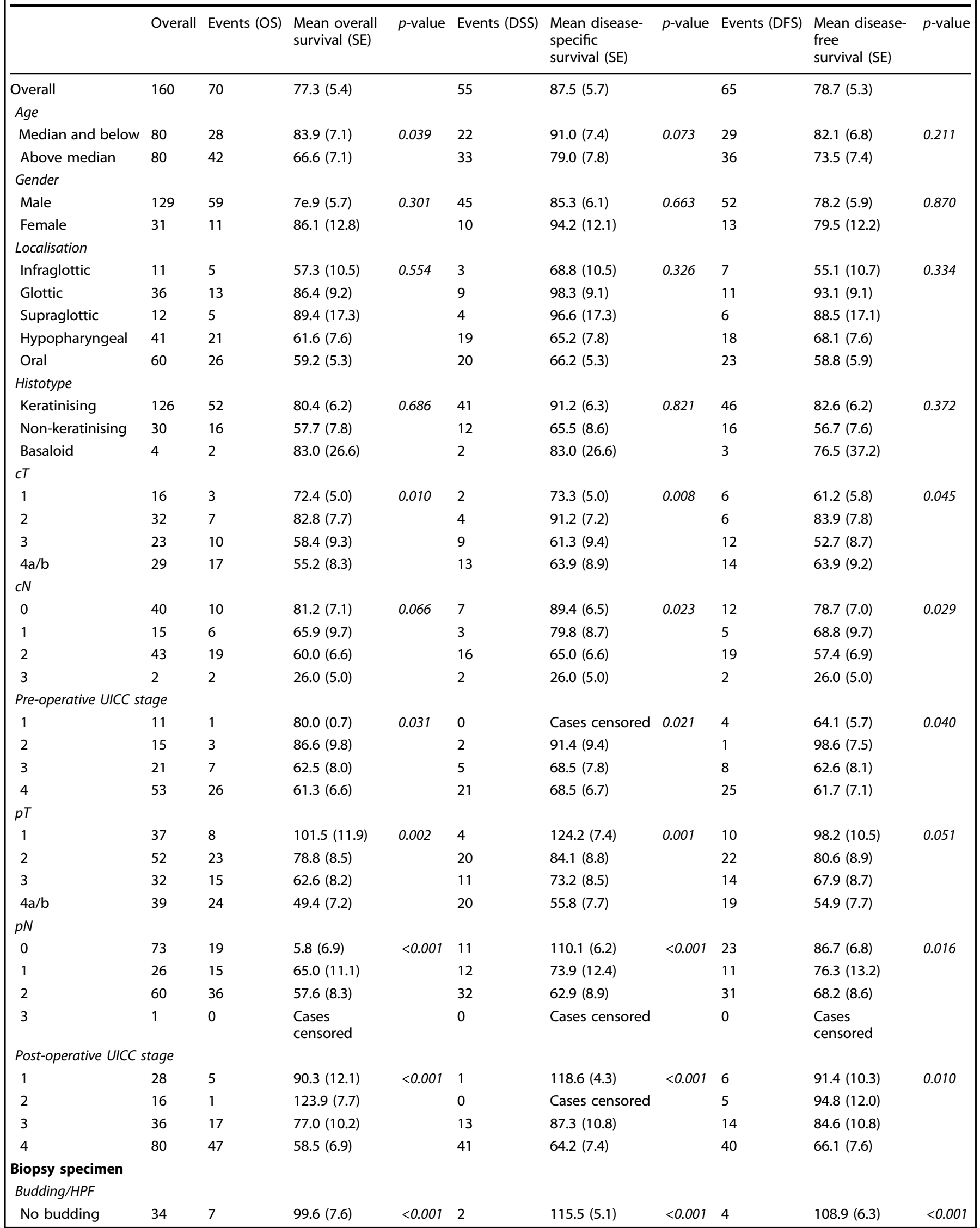




\begin{tabular}{|c|c|c|c|c|c|c|c|c|c|c|}
\hline & Overall & Events (OS) & $\begin{array}{l}\text { Mean overall } \\
\text { survival (SE) }\end{array}$ & $p$-value & Events (DSS) & $\begin{array}{l}\text { Mean disease- } \\
\text { specific } \\
\text { survival (SE) }\end{array}$ & $p$-value & Events (DFS) & $\begin{array}{l}\text { Mean disease- } \\
\text { free } \\
\text { survival (SE) }\end{array}$ & $p$-value \\
\hline $\begin{array}{l}\text { Low budding } \\
\text { activity }\end{array}$ & 88 & 38 & $73.5(7.7)$ & & 30 & $82.8(8.4)$ & & 40 & $69.6(7.0)$ & \\
\hline $\begin{array}{l}\text { High budding } \\
\text { activity }\end{array}$ & 38 & 25 & $50.7(7.7)$ & & 23 & $53.3(7.9)$ & & 21 & $53.5(7.9)$ & \\
\hline \multicolumn{11}{|l|}{ Cell nest size } \\
\hline Large cell nests & 16 & 4 & $89.7(12.8)$ & 0.004 & 1 & $111.9(10.3)$ & $<0.001$ & 2 & $102.4(12.2)$ & 0.001 \\
\hline $\begin{array}{l}\text { Intermediate } \\
\text { cell nests }\end{array}$ & 18 & 3 & $97.9(8.4)$ & & 1 & $108.3(5.5)$ & & 2 & $106.7(5.6)$ & \\
\hline Small cell nests & 55 & 24 & $76.7(9.6)$ & & 19 & $86.8(10.3)$ & & 26 & $66.7(8.4)$ & \\
\hline $\begin{array}{l}\text { Single-cell } \\
\text { invasion }\end{array}$ & 71 & 39 & $59.2(7.2)$ & & 34 & $64.8(7.8)$ & & 35 & $69.2(8.0)$ & \\
\hline 5 & 52 & 23 & $76.3(9.8)$ & & 19 & $84.7(10.3)$ & & 25 & $66.7(8.6)$ & \\
\hline 6 & 37 & 15 & $70.7(11.3)$ & & 11 & $81.7(12,8)$ & & 16 & $73.6(11.6)$ & \\
\hline 7 & 37 & 25 & $48.8(7.7)$ & & 23 & $51.4(8.0)$ & & 20 & $55.0(8.1)$ & \\
\hline \multicolumn{11}{|c|}{ Cellular dissociation grade } \\
\hline nG1 & 34 & 7 & $99.6(7.6)$ & $<0.001$ & 2 & $115.5(5.4)$ & $<0.001$ & 4 & $108.9(6.3)$ & $<0.001$ \\
\hline $\mathrm{nG} 2$ & 89 & 38 & $74.0(7.7)$ & & 30 & $83.3(8.4)$ & & 41 & $69.0(6.9)$ & \\
\hline nG3 & 37 & 25 & $48.8(7.7)$ & & 23 & $51.4(.0)$ & & 20 & $55.0(8.1)$ & \\
\hline \multicolumn{11}{|c|}{ Resection specimen } \\
\hline \multicolumn{11}{|l|}{ Budding/10 HPF } \\
\hline No budding & 31 & 6 & $101.2(7.8)$ & $<0.001$ & 1 & $119.5(3.5)$ & $<0.001$ & 3 & $112.5(5.4)$ & $<0.001$ \\
\hline Small cell nests & 45 & 16 & $82.2(11.3)$ & & 13 & $93.4(10.9)$ & & 19 & $73.9(10.3)$ & \\
\hline $\begin{array}{l}\text { Single-cell } \\
\text { invasion }\end{array}$ & 83 & 48 & $54.2(5.4)$ & & 41 & $59.4(5.9)$ & & 43 & $56.2(5.2)$ & \\
\hline \multicolumn{11}{|l|}{ Sum score } \\
\hline 2 & 6 & 1 & $67.0(8.0)$ & 0.001 & 0 & Cases censored & $<0.001$ & 1 & $72.0(2.8)$ & $<0.001$ \\
\hline 3 & 25 & 5 & $101.3(8.6)$ & & 1 & $118.6(4.3)$ & & 2 & $115.2(5.1)$ & \\
\hline 5 & 45 & 16 & $84.1(11.0)$ & & 13 & $94.5(10.8)$ & & 18 & $78.0(10.4)$ & \\
\hline 6 & 41 & 19 & $52.0(4.4)$ & & 14 & $57.4(4.5)$ & & 19 & $51.7(4.2)$ & \\
\hline 7 & 43 & 29 & $48.1(7.0)$ & & 27 & $50.3(7.2)$ & & 25 & $51.9(7.1)$ & \\
\hline \multicolumn{11}{|c|}{ Cellular dissociation grade } \\
\hline nG1 & 31 & 6 & $101.2(7.8)$ & $<0.001$ & 1 & $119.5(3.5)$ & $<0.001$ & 3 & $112.5(5.4)$ & $<0.001$ \\
\hline $\mathrm{nG} 2$ & 86 & 35 & $77.0(8.3)$ & & 27 & $87.4(9.0)$ & & 37 & $71.8(7.5)$ & \\
\hline nG3 & 43 & 29 & $48.1(7.0)$ & & 27 & $50.3(7.2)$ & & 25 & $51.9(7.1)$ & \\
\hline
\end{tabular}

in other squamous cell carcinomas entities like oesophageal or cervical SCC but was also recently demonstrated to work nicely on pre-therapeutic biopsies of oesophageal SCC.

Biopsies are the mainstay of pre-operative diagnostics in many cancer types, including HNSCC. Pre-therapeutic biopsies are not only necessary to establish a diagnosis but offer the possibility to determine histological subtypes, differentiation and potential further prognostic/predictive information to identify biologically aggressive tumours or to predict treatment response. Therewith, histopathological grading in biopsy specimen guides choice of therapies, including e.g., neoadjuvant systemic treatment versus primary resection or watchful waiting strategies in entities as endometrial cancer and prostate cancer. ${ }^{18,19}$ However, up to date, pre-operative grading in HNSCC is almost meaningless as 
Table 2. Correlation of histomorphological parameters and cellular dissociation grade with clinicopathological data.

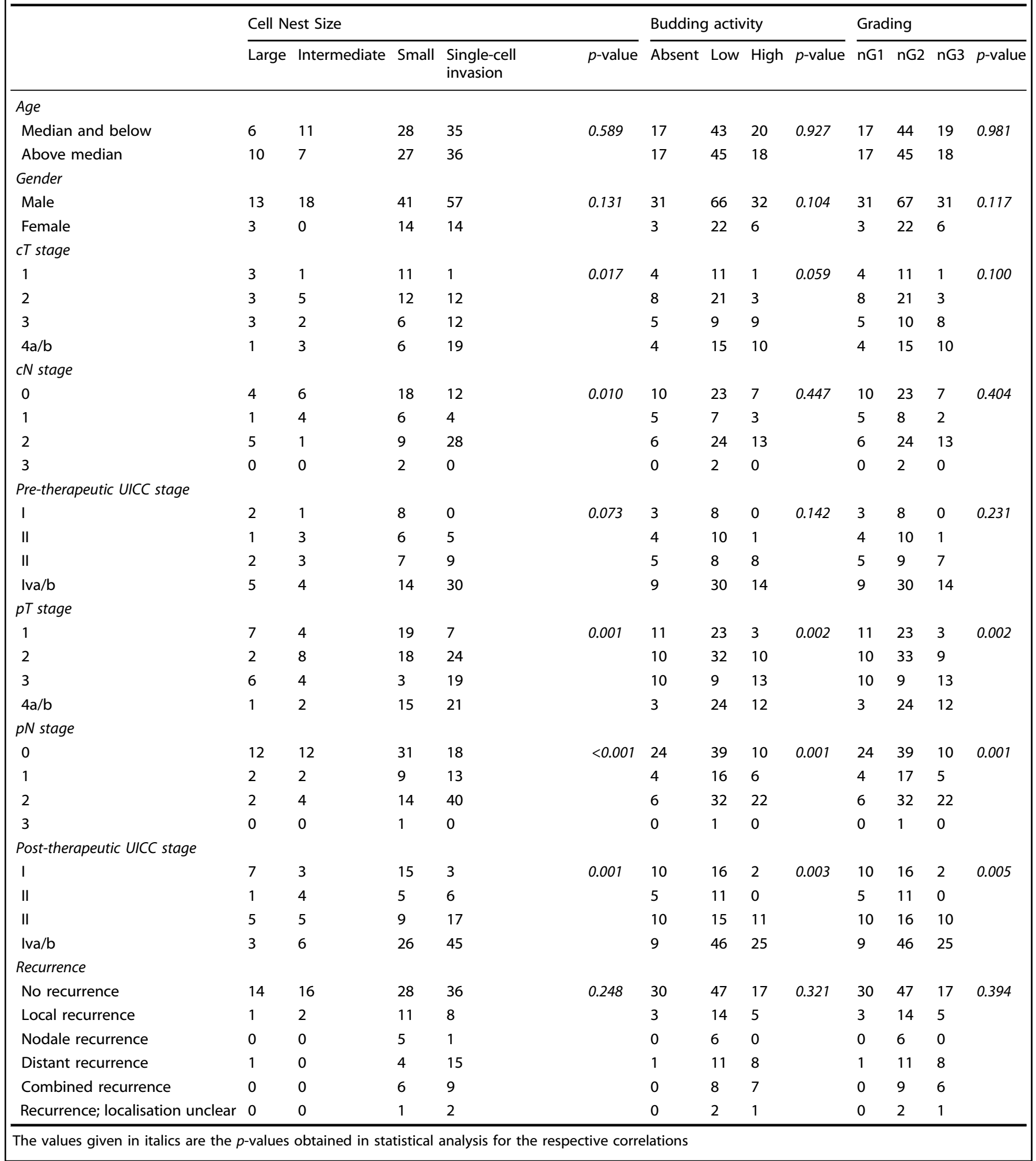

conventional grading according to the $\mathrm{WHO}$ is neither correlated with post-operative tumour stage nor with patient outcome, ${ }^{13-17}$ and is also known to be highly discordant when biopsies and corresponding resection specimen are compared. ${ }^{20,21}$

Based on the above said, major questions remain. Can the high prognostic value of CDG be transferred to small biopsy specimens in HNSCC without losing its prognostic impact concerning patient survival? Can CDG potentially serve as a biomarker for prediction of occult metastasis in cNO necks in clinically early-stage HNSCC? How is the concordance of CDG when biopsies and corresponding resection specimens are compared? Aiming to answer these questions, we investigated Tumour Budding and Cell Nest Size 
and CDG derived from these factors in pre-operative biopsies and resection specimen of $n=160$ patients with HNSCC located in the oral cavity, larynx and hypopharynx. We analysed concordance of CDG between biopsy and resection specimens and correlated CDG with pre-operative clinical staging $(\mathrm{cT}, \mathrm{cN})$, post-operative pathological staging $(\mathrm{pT}, \mathrm{pN})$ and with patient outcome (overall (OS), disease-specific (DSS) and disease-free survival (DFS)).

\section{METHODS}

Patient cohort

Included in our study were 160 patients with HNSCC of the larynx (supraglottic, glottic, infraglottic primaries), the hypopharynx and the oral cavity from whom pre-operative biopsies and resection specimen were prospectively collected. All patients were treated in curative intent between 2004 and 2015 at Klinikum Rechts der Isar of the Technical University of Munich, Germany. All patients received a complete surgical resection of their primary tumours followed by a neck dissection in combination with radiotherapy (65-70 Gy) or radio-chemotherapy if indicated (pT4 tumours and/ or nodal stage pN1 and pN2a/b) according to German guidelines. Exclusion criteria were distant metastasis at the time of diagnosis (cM1 stage), neoadjuvant treatment with radio- and/or chemotherapy and pathological diagnosis upon fresh-frozen tissue sections. Male patients (129/160 (80.6\%)) were in the majority compared with female counterparts $(31 / 160(19.4 \%))$. Mean age at diagnosis was 60.6 years (range: $40.0-87.0$ years). Mean follow-up time of patients alive at the endpoint of analysis was 53.5 months, mean survival time of deceased patients 26.9 months. During follow-up, 70/160 (43.8\%) of patients died and 65/160 (40.6\%) suffered from (local, nodal and/or distant) relapse. Pre-operative clinical staging was performed by clinical examination, ultrasound and/or CT-scan according to the German guidelines. ${ }^{22}$ Clinical and pathological T-stage, N-stage and UICC stage, which were available for $100 / 160(62.5 \%)$, were documented according to the TNM classification of malignant tumours. ${ }^{23}$ Distribution of clinicopathological parameters is given in Tables 1 and 2. Approval for the study was obtained from the Ethics Review Committee of the Technical University of Munich (296/17s).

Histopathological evaluation

Biopsy and resection specimen from all 160 patients were available. Full block haematoxylin and eosin (H\&E)-stained slides of biopsy specimen were evaluated simultaneously by experienced pathologists (MB., MJ.) blinded to clinicopathological data and follow-up using an Olympus BX43 microscope with a field diameter of $0.55 \mathrm{~mm}\left(0.24 \mathrm{~mm}^{2}\right)$. Resection specimen had previously been evaluated in the context of two recently published studies. ${ }^{17,24}$ In case of discordance between biopsy and resection specimen, both biopsy and resection specimen were comparatively re-evaluated. HNSCC were categorised according to the current WHO classification criteria of tumours of head and neck into non-keratinising, keratinising and basaloid. ${ }^{3}$

Tumour Budding and Cell Nest Size and subsequent CDG were evaluated as described before in detail. ${ }^{17,24,25}$ In short, Tumour Budding was defined as "branching" of small tumour nests harbouring $<5$ tumour cells into the stroma/parenchyma, ${ }^{17,26,27}$ and was assessed in one high-power-field (HPF) in biopsies and in ten (continuous) HPFs in resection specimen in areas showing maximal budding activity. The slight modification of the evaluated tumour area (one HPF in biopsies versus ten HPFs in resection specimen) was necessary due to the limited tissue availability in biopsy samples. Low budding activity was defined as 1-4 buds in one HPF (biopsies) and as 1-14 budding nests in ten HPFs (resections), high budding activity was defined as $\geq 5$ in one HPF (biopsies) and as $\geq 15$ budding nests in ten HPFs (resections). Overall Cell Nest Size, a parameter additionally measuring the dissociative growth capability of an individual tumour from a qualitative point of view, was evaluated in tumour area containing smallest tumour cell nests. Tumour cell nests, defined as clustered tumour cells surrounded by tumour stroma, were classified according the size of the smallest invasive cell nest as follows: > 15 tumour cells = large nests, 5-15 tumour cells = intermediate nests, 2-4 tumour cells = small nests, discohesive tumour cells without nested architecture = single-cell invasion. The criteria for budding activity and cell nest size evaluation applied were similar to already established algorithms for the evaluation of CDG in biopsies and resection specimen. According to our previously proposed grading system, ${ }^{17,24,25}$ scores for budding activity $(1=$ no budding activity; 2 =low budding activity; 3 = high budding activity) and cell nest size ( $1=$ large cell nests; $2=$ intermediate cell nests; $3=$ small cell nests; $4=$ single-cell invasion) were assigned in order to obtain the CDG. After summarising both scores into a sum score from 2 to 7, HNSCC were graded into well cohesive (CDG nG1; sum scores 2,3), moderately cohesive (CDG nG2; sum scores 4-6) and poorly cohesive (CDG nG3; sum score 7) according to the CDG.

\section{Statistics}

Associations of grouped morphological characteristics with clinicopathological parameters were calculated with $\mathrm{X}^{2}$ test as well as $X^{2}$ test for trends and Fisher's exact test. Cohen's kappa was used to assess concordance between clinical and pathological staging parameters as well as to assess concordance between histopathological parameters in biopsies and resection specimen. Survival probabilities were plotted with the Kaplan-Meier method, a log-rank test was used to probe for the significance of differences in survival. Multivariate survival analysis was performed with the Cox proportional hazard model. $P$-values $\leq 0.05$ were considered significant. All statistical tests were performed two-sided, and a significance level of $5 \%$ was used.

\section{RESULTS}

Tumour Budding and Cell Nest Size in pre-therapeutic biopsy specimens

The majority of HNSCC were keratinising tumours (126/160, $78.8 \%)$, whereas non-keratinising cancers (30/160, 18.8\%) and basaloid cancers $(4 / 160 ; 2.4 \%)$ were less frequent. No tumour budding activity (per HPF) was detected in $34 / 160$ (21.3\%), while low budding activity was observed in $88 / 160$ (55.0\%) of cases and high budding activity in $38 / 160$ (23.7\%) of cases. Analysis of the Cell Nest Size revealed large cell nests in $16 / 160$ cases $(10.0 \%)$, intermediate nests in $18 / 160$ cases $(11.3 \%)$ and small cell nests in $55 / 160$ cases $(34.3 \%)$. In total, $71 / 160$ cases $(44.4 \%)$ showed infiltrative singular tumour cells, and were therefore classified as single-cell invasion. Addition of scores obtained for budding activity (scores 1-3) and cell nest size (scores 1-4) resulted in a sum scores 2-7 for each case, which was assigned to the final CDG ( $\mathrm{nG} 1, \mathrm{nG} 2, \mathrm{nG} 3$ ) as described in the Methods section. While 34/160 (21.3\%) HNSCC were well differentiated (nG1), 89/160 (55.6\%) were moderately differentiated (nG2) and 37/160 (23.1\%) poorly differentiated (nG3; Table 1; Fig. 1) according to the CDG.

Concordance of the Cellular Dissociation Grade between pretherapeutic biopsies and resection specimens

The resection specimen of this cohort have previously been evaluated in two studies applying a similar grading algorithm. ${ }^{17,24}$ Comparison of Tumour Budding in the corresponding biopsies and resection specimen revealed a high concordance with similar scores obtained in 147/160 (91.9\%) cases. The corresponding Kappa value was $\mathrm{K}=0.87$. Concerning Cell Nest Size, concordance rate was considerably lower with 102/160 (63.8\%) HNSCC showing the same cell nest size in biopsy and resection specimen (Kappa value $\mathrm{K}=0.44)$. Analysis of concordance rate between assigned grades resulted in $147 / 160$ (93.1\%) HNSCC with identical 

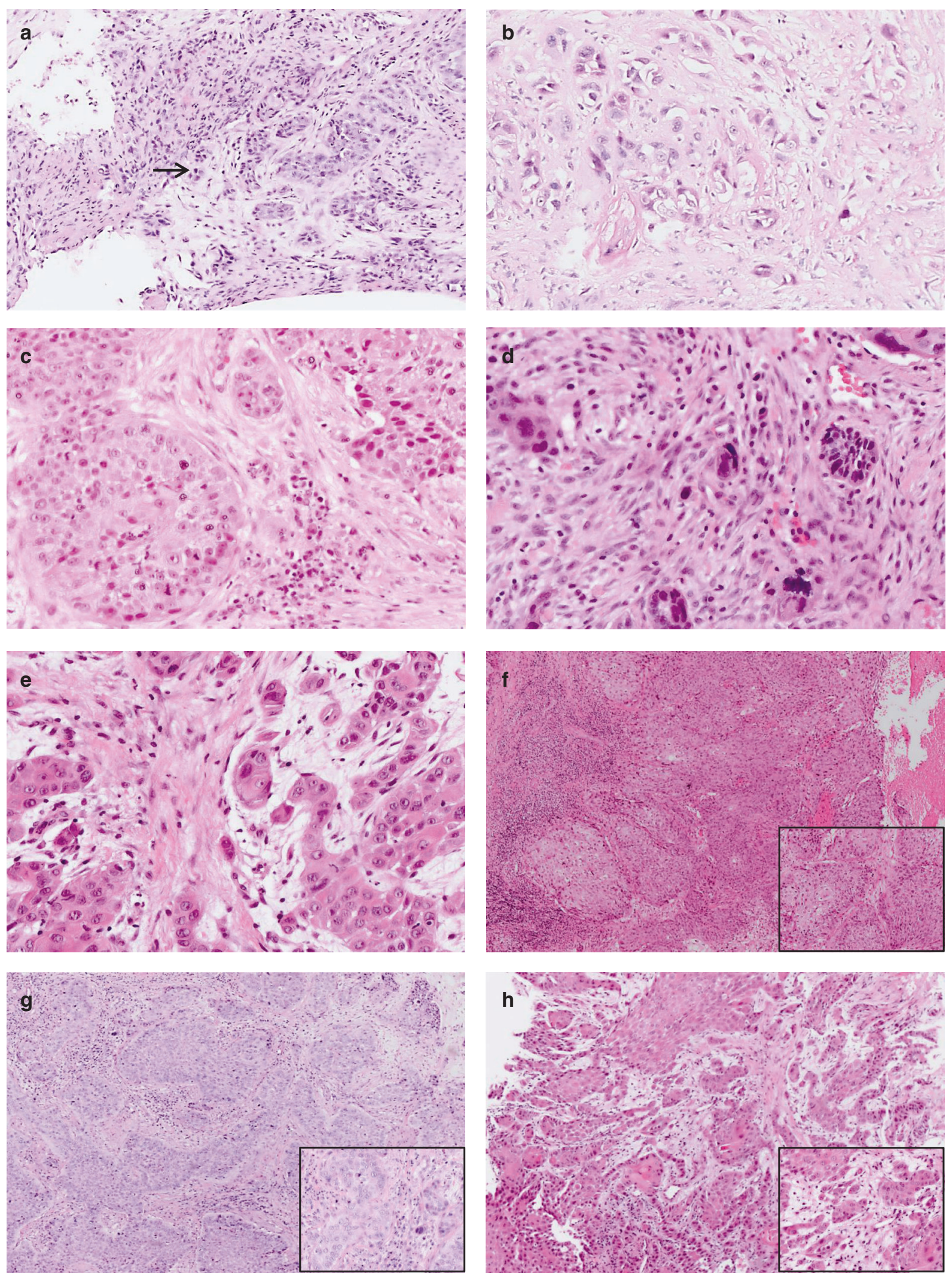

histopathological grade (Kappa value $K=0.89$ ). Of the 13 cases with discordant grading, which was confirmed upon re-evaluation of biopsy and resection specimen, 12 showed an upgrading whereas only one case was downgraded from $n G 2$ to $n G 1$
(Supplementary Table 1). Upgrading was observed in the setting of very small tumour-containing biopsy specimen $(<0.3 \mathrm{~cm})$. Downgrading was detected in a large biopsy specimen of a pT1 carcinoma, which contained a majority of the whole tumour 
Fig. 1 Representative H\&E stained biopsy specimen of head and neck squamous cell carcinomas (HNSCC). a depicts a HNSCC with low Tumour Budding as indicated by presence of $<5$ cell nests containing $<5$ tumour cells within one HPF ( $\rightarrow$ highlighting the two tumour buds), b depicts a HNSCC with high Tumour Budding as indicated by presence of $\geq 5$ cell nests containing $<5$ tumour cells within one HPF $\left(\rightarrow\right.$ highlighting some of the numerous tumour buds of this carcinoma). c-e depict different Cell Nest Sizes with $\mathbf{C}$ showing large $\left(^{*}\right)$ and intermediate $(\rightarrow)$ cell nest sizes, while $\mathbf{d}+\mathbf{e}$ show carcinomas with small cell nests $\left({ }^{*}\right)$ and single-cell invasion $(\rightarrow)$. f-h Overview over representative HNSCC biopsies with $\mathbf{f}$ showing Cellular Dissociation Grade nG1 carcinoma (no Tumour Budding, large Cell Nest Size), g showing a Cellular Dissociation Grade nG2 carcinoma (low Tumour Budding, small Cell Nest Size) and $\mathbf{h}$ showing a Cellular Dissociation Grade nG3 carcinoma (high Tumour Budding, single-cell invasion). Inserts showing the HPF[ $\times 40]$ of the respective cases with the highest Tumour Budding activity and the smallest Cell Nest Size within the biopsy specimen.

leaving only small remnants of the invasive tumour within the resection specimen.

Correlation of Tumour Budding, Cell Nest Size and Cellular Dissociation Grade in biopsies with pre-operative staging

Cell nest size was significantly correlated with $\mathrm{cT}(p=0.017)$ and cN $(p=0.010)$ stage with single-cell invasion being more frequently detected in HNSCC patients with higher clinical stages. No further significant correlation of either budding activity or overall grading with clinical staging parameters were observed (Table 2).

Correlation of Tumour Budding, Cell Nest Size and Cellular Dissociation Grade in biopsies with post-operative staging Cell Nest Size and Tumour Budding as well CDG were significantly correlated with post-operative pathological staging parameters $\mathrm{pT}$ stage, pN stage and UICC stage. Presence of small cell nest sizes and single-cell invasion as well as presence of budding activity were more frequently detected in cases with higher $\mathrm{pT}, \mathrm{pN}$ and UICC stage $(p<0.005)$. This was mirrored by correlation of moderate ( $\mathrm{nG} 2$ ) and poor (nG3) differentiation with higher postoperative stages ( $p<0.01$; Table 2, Fig. 2).

Concordance of pre-therapeutic and post-therapeutic staging parameters

Clinical CT and post-operative pT stage was discordant in $36 / 100$ (36.0\%) HNSCC (Kappa value $\mathrm{K}=0.52$ ). In total, 14 cases received an upstaging and 22 a downstaging after pathological evaluation of the resection specimen. Respective values for nodal stage and UICC stage were as follows: discordance rate cN vs. pN 32/100 (32.0\%; $\mathrm{K}=0.49)$; pre-operative UICC vs. post-operative UICC 33/ $100(33.0 \% ; \mathrm{K}=0.49)$. Upstaging: nodal stage 12 cases; UICC stage 14 cases. Downstaging: nodal stage 20 cases; UICC stage 19 cases (Supplementary Table 2).

Subgroup analysis of HNSCC with clinically nodal-negative (cN0) necks: correlation of Cellular Dissociation Grade in biopsies with post-operative staging parameters

HNSCC patients with nodal-negative cervical lymph nodes upon clinical staging procedures (cN0 necks; $n=40$ ) showed occult metastases detected by pathological evaluation of neck dissection specimen in $8 / 40(20.0 \%)$ cases. Histopathological grading of all of these cases was $n G 2 / 3$, whereas in none of the $n G 1$ cases presence of lymph node metastases was observed. This result indicated a positive predictive value PPV $=100 \%$ for $n G 1$ grading to predict nodal negativity upon pathological work-up in cNO necks. Association of grading with post-operative nodal stage is summarised in Supplementary Table 3.

Correlation of Tumour Budding, Cell Nest Size and Cellular Dissociation Grade in biopsies with patient survival

Tumour budding activity was strongly associated with decreased OS, DSS and DFS ( $p<0.001$, respectively) with HNSCC with high budding activity revealing the shortest mean OS. In analogy, Cell Nest Size was strongly correlated with OS, DSS and DFS $(p<0.01$, respectively). Cases with single-cell invasion and small cell nests suffered from earlier deaths and tumour recurrences compared with their counterparts (Table 1; Supplementary Fig. 1). Not only both morphologic patterns but also the obtained sum score was significantly correlated with survival parameters of patients $(p<0.005$; Table 1$)$.

Consequently, the CDG on pre-therapeutic biopsies which is based on the sum scores also showed a highly significant impact on OS, DSS and DFS ( $p<0.001$, respectively) (Table 1, Fig. 3 ). While mean DSS of nG1 tumours was 119.5 months, it dropped to 87.4 months for nG2 HNSCC and to 50.3 months for nG3 HNSCC (Table 1).

In order to prove that the prognostic impact of the novel grading system derived from biopsy specimens is independent from clinicopathological data and tumour staging, we performed a multivariate analysis by applying a cox regression model incorporating pre-operative, respectively, post-operative staging parameters (cUICC/pUICC), gender and age and the CDG. Multivariate analysis confirmed the independent prognostic value of the novel grading concerning patient survival (OS, DSS, DFS; Table 3 for cox regression with pUICC; Supplementary Table 4 for cox regression with CUICC). Hazard ratio for DSS was 6.1 (95\% confidence interval 1.4-26.4) for nG2 and 11.1 (95\% confidence interval 2.6-48.0) for $\mathrm{nG} 3$ HNSCC compared to $\mathrm{nG} 1$ cases ( $p=$ 0.002; multivariate analysis including pUICC).

\section{DISCUSSION}

Pre-operative biopsy is the gold standard for cancer diagnosis in many cancers, including HNSCC. Ideally, histomorphologic diagnosis and subsequent histopathologic grading upon biopsy enables a prognostic patient stratification by distinction of cancers with comparably benign clinical course from those with aggressive tumour biology. In many entities including prostate and endometrial cancer, pre-operative biopsies therewith enable guidance of therapeutic approaches and clinical decision making, including the choice for neoadjuvant treatments or the selection of watch and wait strategies. ${ }^{18,19}$ In contrast, studies in HNSCC revealed, that conventional histopathologic grading as defined by WHO criteria in incisional biopsies lacks impact on clinical outcome, ${ }^{20,21}$ which is in line with studies that reported WHO grading to be of only limited prognostic relevance in resection specimen. ${ }^{13-17}$ Another important pre-exquisite-if therapy decisions should be based on biopsy readouts-is to ensure the representativity of the data which can be obtained from biopsies. Thus, a high concordance of grading in biopsies and corresponding resection specimen is desirable, as discordances can potentially lead to erroneous decisions and treatment failure. Current WHO grading in HNSCC biopsies not only has limited prognostic impact but also fails to meet this criterion as Dik et al. recently found major discordance of grading between biopsy and corresponding resection specimen for this scheme. ${ }^{20,21}$

We recently proposed a novel grading algorithm in HNSCC with high prognostic impact ${ }^{17,24}$ and high inter- and intra-observer concordance $^{25}$ termed Cellular Dissociation Grade, which has also been shown to work nicely in other SCC entities including in pretherapeutic biopsies of oesophageal SCC. The CDG is based on the cellular dissociation parameters Tumour Budding and Cell Nest Size, which are both histomorphologic readouts measuring the 

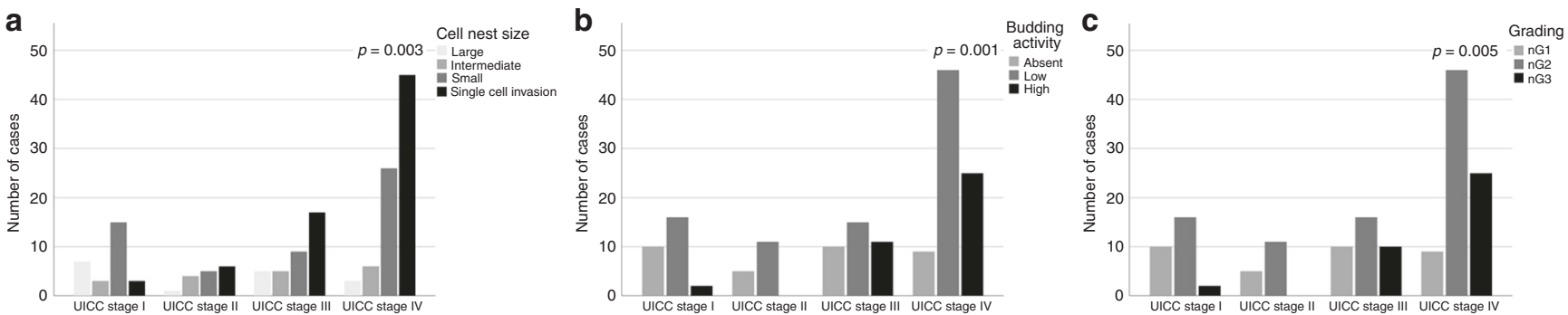

Fig. 2 Associations of UICC stage with histomorphological parameters and Cellular Dissociation Grade. Association between UICC tumour stage with Cell Nest Size (a), Tumour Budding (b) and Cellular Dissociation Grading (c).

capability of dissociative growth of an individual carcinoma from either a quantitative (Tumour Budding) or qualitative (Cell Nest Size) point of view. Considering the above discussed potential major prognostic and predictive implications of the data derived early in the diagnostic process from biopsy specimens, we aimed to probe if the prognostic value of the CDG is retained when assessed in pre-therapeutic biopsies of patients suffering from HNSCC. In addition, we analysed the value of the pre-operative CDG for the prediction of post-operative tumour stage, including the presence of occult lymph node metastasis in clinically and radiologically negative cNO necks.

We found, that Tumour Budding and Cell Nest Size are of major prognostic relevance for patient outcome with presence of high budding activity and single-cell invasion indicating the most aggressive disease course with early relapses and poor survival. Summarising these two parameters and applying our CDG (nG1, nG1, nG3) discriminated three distinct prognostic groups. The prognostic value of the grading system was independent of potential additional influencing factors, such as age, gender and stage and revealed hazard ratios for OS (DSS; DFS) for $n G 2 / n G 3$ HNSCC of $2.5(6.1 ; 5.0) / 4.2(11.1 ; 7.4)$. Again, previous studies revealed that the current conventional WHO-based grading of HNSCC does not show prognostic impact when applied in biopsy specimens. $^{20,21}$ In addition, the Kappa value $\mathrm{K}=0.89$ for the corresponding biopsy and resection specimen grading with $94 \%$ concordantly graded HNSCC proves the representativity of the biopsy grading results for the overall tumour configuration. Of 160 HNSCC, an upgrading from biopsy to resection specimen was only observed in ten cases. In all of these cases, the biopsies only harboured very superficially captured carcinoma. In only one case, downgrading was evident. Review of both specimens revealed that this case was a T1 HNSCC, in which a large incisional biopsy comprised the major part of the carcinoma leaving only residual invasive cancer within the resection specimen. Regarding the concordance of the two parameters budding and cell nest size in biopsy and the corresponding resection specimen, concordance rate of the morphologic pattern tumour budding was strikingly higher $(\kappa=0.87)$ compared with the pattern cell nest size $(\kappa=$ 0.44). While tumour budding provides an overview of the dissociative growth capability, cell nest size represents the highest dissociative capability within the area of highest aggressiveness. This might be the reason why tumour heterogeneity plays a more prominent role in the evaluation of the cell nest size: biopsies by nature only capture a smaller part of carcinomas compared to resection specimen, potentially leading to a bias induced by tumour heterogeneity when evaluating cell nest size as the area of highest dissociative capability might not be included in the biopsy specimen. However, this bias particularly comes to effect in cases with scores $2 / 3$ for cell nest size, and therewith obviously does not lead to a major effect on the sum score and the final grade, which is demonstrated by the high kappa value for the overall grading. Dik et al. analysed concordance of WHO-based grading of HNSCC between biopsy and resection specimens and found $>50 \%$ discordantly graded cases in their studies. ${ }^{20,21}$ Concordance rates of histopathological grading in other entities as prostate cancer were higher with $50-70 \%$ concordant cases. ${ }^{28,29}$ The concordance rate of our grading is surprisingly high, which might be due to large and representative incisional biopsies performed at our institution.

Besides our grading approach, several alternative HNSCC grading systems have been proposed, including the "malignancy grading of invasive margins" (MG), ${ }^{30}$ the "histological risk model" $(\mathrm{HR})^{15}$ and the "tumour budding and depth of invasion" $(\mathrm{BD})^{31}$ grading system. In contrast to our CDG, which has been successfully applied in oral $\mathrm{SCC}^{17,25}$ in laryngeal and in hypopharyngeal $\mathrm{SCC}_{1}{ }^{24}$ and which has been transvalidated in oesophageal, ${ }^{26}$ cervical $^{32}$ and pulmonary $\mathrm{SCC}_{1}{ }^{27}$ all of these approaches yielded varying results with respect to prognostic value and interobserver variability. ${ }^{15,16,30,31,33-37}$ Only the prognostic impact of the BD grading system, which evaluates BA together with tumour invasion depth, was confirmed in recent studies. ${ }^{16,31}$ However, per definition tumour invasion depth is a parameter that rather describes tumour stage than tumour grade as defined by the current TNM manual. ${ }^{23,38,39}$ Furthermore, this parameter is not applicable in biopsies, limiting its value for routine biopsy diagnostics. ${ }^{38,40}$

Incorporation of markers of cellular dissociative capability such as Tumour Budding and Cell Nest Size into a grading system seems reasonable as presence of Tumour Budding has been shown to be associated with an unfavourable disease course not only in HNSCC (reviewed in refs. ${ }^{41,42}$ ) but also in other solid tumours, such as colorectal ${ }^{43,44}$ and pulmonary carcinomas. ${ }^{27}$ Confirming our results, Tumour Budding has been shown to be of prognostic value not only in resection but as well in biopsy specimen of these tumour entities (reviewed in ref. ${ }^{45}$ ). Focusing on the biologic background of Tumour budding, recent research has shown, that this histologic pattern might be the morphologic representative of epithelial-mesenchymal transition (reviewed in ref. ${ }^{46}$ ). It has been identified as a sign of invasion and metastasis and has therefore been proposed as a novel hallmark of cancer by Makitie et al. (reviewed in ref. ${ }^{47}$ ).

Both, Tumour Budding and Cell Nest Size as well as the composite parameter CDG when assessed in pre-operative biopsies were strongly correlated with post-operative $\mathrm{pT}$ and $\mathrm{pN}$ stage. Therefore, post-operative positive nodes were significantly more frequent in cases with a moderate or poor tumour differentiation ( $\mathrm{nG} 2 / \mathrm{nG} 3$ ) according to the CDG compared with well-differentiated carcinomas. In contrast, only Cell Nest Size but not Tumour Budding or CDG was correlated with pre-operative staging parameters $(\mathrm{cT} / \mathrm{cN})$. This might be due to the high discordance rate of pre-operative clinical $\mathrm{CT}$ and $\mathrm{CN}$ stage compared with post-operative $\mathrm{pT}$ and $\mathrm{pN}$ in $36 \%$, respectively, $32 \%$ cases - a finding which confirms previous studies in HNSCC studies showing comparably low concordance rates between clinical and pathological staging. ${ }^{6,48,49}$ Furthermore, in our study, the subgroup of clinically early HNSCC in UICC stages I and II $20 \%$ of cNO necks revealed occult nodal metastasis only detected by pathological examination. Considering the ongoing discussion if 
a
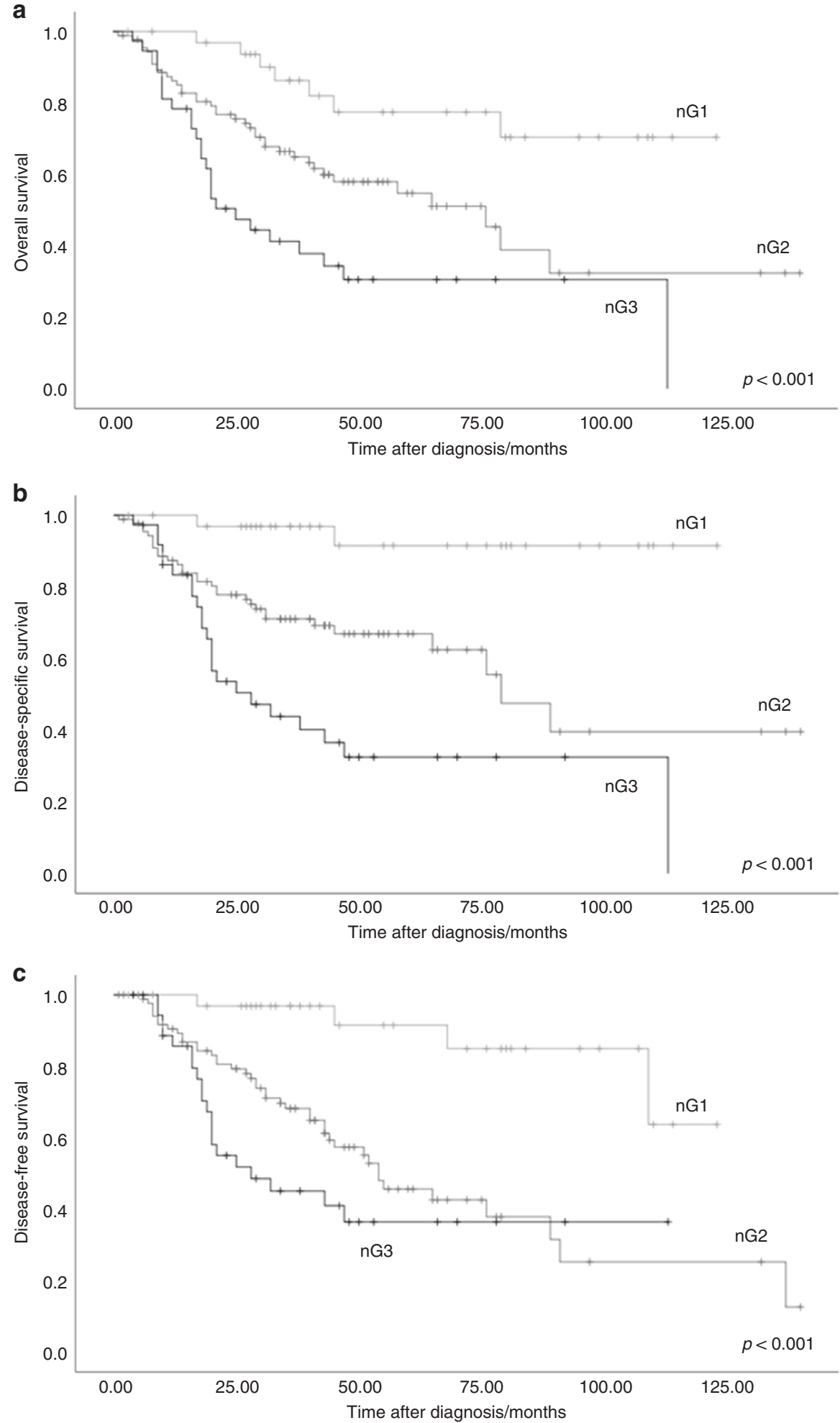

Fig. 3 Association of Cellular Dissociation Grade with survival. a Overall survival, b disease-specific survival, c disease-specific survival.

clinically nodal-negative necks should be treated by elective neck dissection or by watchful waiting strategies, these results support the notion and confirm previous studies that clinical staging in its current form is not a valuable predictive tool for treatment planning in these patients, ${ }^{10,11,50,51}$ as it lacks the necessary sensitivity for detection of metastatic disease. Searching for a better biomarker for the presence of occult nodal metastasis in these patients, Sakata et al. proposed Tumour Budding as a novel predictor of occult lymph node metastasis in oral $\mathrm{SCC}^{12}$ in a recent study. In our study-confirming this result-none of clinically cNOstaged patients with nG1 tumour differentiation according to the CDG revealed an occult nodal metastasis upon pathological workup, whereas occult metastasis was detected in $36 \%$ of $n G 2 / n G 3$ early-stage patients. Our study therewith underlines the potential 
Table 3. Multivariate survival analysis including Cellular Dissociation Grade, age, gender and post-operative UICC stage.

\begin{tabular}{|c|c|c|c|c|}
\hline & \multirow{2}{*}{$\begin{array}{l}\text { Hazard ratio } \\
\text { HR }\end{array}$} & \multicolumn{2}{|c|}{$\begin{array}{l}\text { Confidence } \\
\text { interval }\end{array}$} & \multirow[t]{2}{*}{$p$-value } \\
\hline & & Low & High & \\
\hline \multicolumn{5}{|l|}{ Overall survival } \\
\hline \multicolumn{5}{|l|}{ Gender } \\
\hline Male & 1.000 & & & 0.160 \\
\hline Female & 0.625 & 0.325 & 1.204 & \\
\hline \multicolumn{5}{|l|}{ Age } \\
\hline Median and below & 1.000 & & & 0.022 \\
\hline Above median & 1.764 & 1.087 & 2.880 & \\
\hline \multicolumn{5}{|l|}{ UICC stage } \\
\hline 1 & 1.000 & & & 0.008 \\
\hline II & 0.298 & 0.034 & 2.574 & \\
\hline III & 2.043 & 0.740 & 5.644 & \\
\hline Iva/b & 3.194 & 1.250 & 8.164 & \\
\hline \multicolumn{5}{|c|}{ Cellular Dissociation Grade } \\
\hline nG1 & 1.000 & & & 0.004 \\
\hline nG2 & 2.500 & 1.078 & 5.797 & \\
\hline nG3 & 4.222 & 1.771 & 10.065 & \\
\hline \multicolumn{5}{|c|}{ Disease-specific survival } \\
\hline \multicolumn{5}{|l|}{ Gender } \\
\hline Male & 1.000 & & & 0.417 \\
\hline Female & 0.750 & 0.375 & 1.502 & \\
\hline \multicolumn{5}{|l|}{ Age } \\
\hline Median and below & 1.000 & & & 0.054 \\
\hline Above median & 1.717 & 0.991 & 2.973 & \\
\hline \multicolumn{5}{|l|}{ UICC stage } \\
\hline 1 & 1.000 & & & 0.046 \\
\hline II & $<0.001$ & $<0.001$ & $>100.0$ & \\
\hline III & 7.462 & 0.962 & 57.875 & \\
\hline Iva/b & 12.382 & 1.690 & 90.713 & \\
\hline \multicolumn{5}{|c|}{ Cellular Dissociation Grade } \\
\hline nG1 & 1.000 & & & 0.002 \\
\hline nG2 & 6.131 & 1.426 & 26.361 & \\
\hline nG3 & 11.095 & 2.565 & 47.999 & \\
\hline \multicolumn{5}{|l|}{ Disease-free survival } \\
\hline \multicolumn{5}{|l|}{ Gender } \\
\hline Male & 1.000 & & & 0.486 \\
\hline Female & 0.802 & 0.431 & 1.492 & \\
\hline \multicolumn{5}{|l|}{ Age } \\
\hline Median and below & 1.000 & & & 0.130 \\
\hline Above median & 1.478 & 0.892 & 2.451 & \\
\hline \multicolumn{5}{|l|}{ UICC stage } \\
\hline I & 1.000 & & & 0.136 \\
\hline II & 1.123 & 0.336 & 3.751 & \\
\hline III & 1.452 & 0.543 & 3.883 & \\
\hline Iva/b & 2.245 & 0.939 & 5.364 & \\
\hline \multicolumn{5}{|c|}{ Cellular Dissociation Grade } \\
\hline nG1 & 1.000 & & & 0.002 \\
\hline nG2 & 5.002 & 1.746 & 14.336 & \\
\hline nG3 & 7.393 & 2.456 & 22.260 & \\
\hline
\end{tabular}

major importance of Tumour Budding as a predictive biomarker for lymph node metastasis in cNO necks. The predictive value could potentially be further increased by combining CDG assessment on biopsies with modern imaging modalities such as PET CT. ${ }^{52}$

Our study clearly has some limitations as the included patient cohort contained a majority of advanced HNSCC. Furthermore, the study cohort included cases from different areas of the head and neck region (oral cavity, larynx, hypopharynx). The study design was based on a prospective-retrospective concept with prospective sample collection from the day of the biopsy/resection followed by retrospective histopathological evaluation. Therefore, additional especially prospective study concepts and multi-centre studies are necessary to validate the prognostic impact of CDG in the pre-operative setting of HNSCC.

Taken together, our study revealed that CDG based on Tumour Budding and Cell Nest Size can successfully be applied in pretherapeutic biopsies of HNSCC. The high prognostic impact of CDG assessed in biopsy specimen, separating three distinct prognostic groups, was independent of other prognostic parameters. Pre-operative grading was furthermore predictive for the presence of occult nodal metastasis, which had not been detected by routine clinical staging procedures in clinically nodal-negative necks. Considering the prognostic impact in the post-therapeutic setting and the high inter- and intra-observer reliability of the CDG which we demonstrated in previous studies, ${ }^{17,25}$ we believe, that grading of HNSCC by CDG shows a promising potential for applicability in daily routine pathological and clinical practice. Taking together our previous studies, ${ }^{17,24,25}$ the major prognostic impact of the CDG was shown in the pre- and post-operative setting in the same homogeneous patient cohort as was the high inter- and intra-observer reproducibility of the grading system. Using the same patient cohort may prove the validity and significance of the results, as it allows to exclude a coincidental effect which might be observed when using different study cohorts.

In conclusion, we believe that our findings provide a rationale for the integration of CDG assessment in routine diagnostics of HNSCC biopsies, since CDG has the potential to play a substantial role in treatment planning and prognostic patient stratification in HNSCC in the pre-therapeutic setting.

\section{ACKNOWLEDGEMENTS}

The authors thank the members of the Munich Biobank for their technical assistance.

\section{AUTHOR CONTRIBUTIONS}

M.J.: data acquisition, data analysis, methodology, study design, project administration and writing —original draft; K.S.: data acquisition and writing —original draft; F.S.: data acquisition and writing-original draft, B.H.: statistical analysis and writingoriginal draft; A.K.: clinical data acquisition and writing-original draft; U.S.: clinical data acquisition and writing-original draft; A.P.: clinical data acquisition and writing —original draft; M.W.: clinical data acquisition and writing —original draft; M.S.: data acquisition and writing - original draft; J.B.: data acquisition, statistical analysis and writing-original draft; A.B.R.: data acquisition and writing-original draft; B.K.: data acquisition and writing-original draft; P.K.: data acquisition and writing-original draft; K.K.: data acquisition and writing-original draft; H.D.: data acquisition and writing-original draft; S.M.: data acquisition and writing-original draft; S.E.C.: data acquisition and writing-original draft; W.W.: methodology, study design, supervision and writing-original draft; M.B.: data acquisition, data analysis, methodology, study design, supervision, project administration and writing-original draft.

\section{ADDITIONAL INFORMATION}

Ethics approval and consent to participate Approval for the study was obtained from the Ethics Review Committee of the Technical University of Munich (296/17 s) and all patients agreed at the beginning of their treatment that their tissue may be used for research purposes. The study was performed in accordance with the Declaration of Helsinki. 
Consent to publish Not applicable.

Data availability All data relevant for this study are given with the main paper, including figures, tables and the supplementary files. The tissue investigated for this study is archived in the Institute of Pathology of the Technical University of Munich

Competing interests The authors declare no competing interests.

Funding information The work was in part funded by the Else Kröner Fresenius Stiftung to Melanie Boxberg.

Supplementary information is available for this paper at https://doi.org/10.1038/ s41416-019-0719-8.

Note This work is published under the standard license to publish agreement. After 12 months the work will become freely available and the license terms will switch to a Creative Commons Attribution 4.0 International (CC BY 4.0).

Publisher's note Springer Nature remains neutral with regard to jurisdictional claims in published maps and institutional affiliations.

\section{REFERENCES}

1. Siegel, R. L., Miller, K. D. \& Jemal, A. Cancer statistics, 2015. CA Cancer J. Clin. 65 5-29 (2015).

2. Marur, S. \& Forastiere, A. A. Head and neck cancer: changing epidemiology, diagnosis, and treatment. Mayo Clin. Proc. 83, 489-501 (2008).

3. El-Naggar, A. K. (Hg.). WHO classification of head and neck tumours: International Agency for Research on Cancer. (2017).

4. Marur, S. \& Forastiere, A. A. Head and neck squamous cell carcinoma: update on epidemiology, diagnosis, and treatment. Mayo Clin. Proc. 91, 386-396 (2016).

5. Pezzuto, F., Buonaguro, L., Caponigro, F., lonna, F., Starita, N., Annunziata, C. et al. Update on head and neck cancer: current knowledge on epidemiology, risk factors, molecular features and novel therapies. Oncology 89, 125-136 (2015).

6. Koch, W. M., Ridge, J. A., Forastiere, A. \& Manola, J. Comparison of clinical and pathological staging in head and neck squamous cell carcinoma: results from Intergroup Study ECOG 4393/RTOG 9614. Arch. Otolaryngol. Head Neck Surg. 135, 851-858 (2009)

7. Leusink, F. K., van Es, R. J., de Bree, R., Baatenburg de Jong, R. J., van Hooff, S. R., Holstege, F. C. et al. Novel diagnostic modalities for assessment of the clinically node-negative neck in oral squamous-cell carcinoma. Lancet Oncol. 13, e554-e561 (2012).

8. Wensing, B. M., Merkx, M. A., De Wilde, P. C., Marres, H. A. \& Van den Hoogen, F. J. Assessment of preoperative ultrasonography of the neck and elective neck dissection in patients with oral squamous cell carcinoma. Oral Oncol. 46, 87-91 (2010).

9. Yuen, A. P., Ho, C. M., Chow, T. L., Tang, L. C., Cheung, W. Y., Ng, R. W. et al. Prospective randomized study of selective neck dissection versus observation for N0 neck of early tongue carcinoma. Head Neck. 31, 765-772 (2009).

10. D'Cruz, A. K., Vaish, R., Kapre, N., Dandekar, M., Gupta, S., Hawaldar, R. et al. Elective versus therapeutic neck dissection in node-negative oral cancer. N. Engl. J. Med. 373, 521-529 (2015).

11. Haddadin, K. J., Soutar, D. S., Oliver, R. J., Webster, M. H., Robertson, A. G. \& MacDonald, D. G. Improved survival for patients with clinically $T 1 / T 2$, N0 tongue tumours undergoing a prophylactic neck dissection. Head Neck. 21, 517-525 (1999).

12. Sakata, J., Yamana, K., Yoshida, R., Matsuoka, Y., Kawahara, K., Arita, H. et al. Tumour budding as a novel predictor of occult metastasis in CT2NO tongue squamous cell carcinoma. Hum. Pathol. 76, 1-8 (2018).

13. Sethi, S., Lu, M., Kapke, A., Benninger, M. S. \& Worsham, M. J. Patient and tumour factors at diagnosis in a multi-ethnic primary head and neck squamous cell carcinoma cohort. J. Surg. Oncol. 99, 104-108 (2009).

14. Bello, I. O., Soini, Y. \& Salo, T. Prognostic evaluation of oral tongue cancer: means, markers and perspectives (II). Oral Oncol. 46, 636-643 (2010).

15. Brandwein-Gensler, M., Teixeira, M. S., Lewis, C. M., Lee, B., Rolnitzky, L., Hille, J. J. et al. Oral squamous cell carcinoma: histologic risk assessment, but not margin status, is strongly predictive of local disease-free and overall survival. Am. J. Surg. Pathol. 29, 167-178 (2005).

16. Sawazaki-Calone, I., Rangel, A., Bueno, A. G., Morais, C. F., Nagai, H. M., Kunz, R. P. et al. The prognostic value of histopathological grading systems in oral squamous cell carcinomas. Oral Dis. 21, 755-761 (2015).

17. Boxberg, M., Jesinghaus, M., Dorfner, C., Mogler, C., Drecoll, E., Warth, A. et al. Tumour budding activity and cell nest size determine patient outcome in oral squamous cell carcinoma: proposal for an adjusted grading system. Histopathology 70, 1125-1137 (2017).

18. Van Praet, C., Libbrecht, L., D'Hondt, F., Decaestecker, K., Fonteyne, V., Verschuere, $S$. et al. Agreement of Gleason score on prostate biopsy and radical prostatectomy specimen: is there improvement with increased number of biopsy cylinders and the 2005 revised Gleason scoring? Clin. Genitourin. Cancer 12, 160-166 (2014).

19. Fischerova, D., Fruhauf, F., Zikan, M., Pinkavova, I., Kocian, R., Dundr, P. et al. Factors affecting sonographic preoperative local staging of endometrial cancer. Ultrasound Obstet. Gynecol. 43, 575-585 (2014).

20. Dik, E. A., Ipenburg, N. A., Kessler, P. A., van Es, R. J. J. \& Willems, S. M. The value of histological grading of biopsy and resection specimens in early stage oral squamous cell carcinomas. J. Craniomaxillofac. Surg. 46, 1001-1006 (2018).

21. Dik, E. A., Ipenburg, N. A., Adriaansens, S. O., Kessler, P. A., van Es, R. J. \& Willems, S. M. Poor correlation of histologic parameters between biopsy and resection specimen in early stage oral squamous cell carcinoma. Am. J. Clin. Pathol. 144, 659-666 (2015).

22. Wolff, K-D., Bikowski, K., Böhme, P., et al. Diagnostik und therapie des mundhöhlenkarzinoms. AWMF Leitlinie (German treatment guidelines, 2012).

23. Gospodarowicz, M. K., Brierley, J. D. \& Wittekind, C. TNM Classification of Malignant Tumours (John Wiley \& Sons, 2017).

24. Boxberg, M., Kuhn, P. H., Reiser, M., Erb, A., Steiger, K., Pickhard, A. et al. Tumour budding and cell nest size are highly prognostic in laryngeal and hypopharyngeal squamous cell carcinoma: further evidence for a unified histopathologic grading system for squamous cell carcinomas of the upper aerodigestive tract. Am. J. Surg. Pathol. 43, 303-313 (2019).

25. Boxberg, M., Bollwein, C., Johrens, K., Kuhn, P. H., Haller, B., Steiger, K. et al. Novel prognostic histopathological grading system in oral squamous cell carcinoma based on tumour budding and cell nest size shows high interobserver and intraobserver concordance. J. Clin. Pathol. 72, 285-294 (2019).

26. Jesinghaus, M., Boxberg, M., Konukiewitz, B., Slotta-Huspenina, J., Schlitter, A. M., Steiger, K. et al. A novel grading system based on tumour budding and cell nest size is a strong predictor of patient outcome in esophageal squamous cell carcinoma. Am. J. Surg. Pathol. 41, 1112-1120 (2017).

27. Weichert, W., Kossakowski, C., Harms, A., Schirmacher, P., Muley, T., Dienemann, H. et al. Proposal of a prognostically relevant grading scheme for pulmonary squamous cell carcinoma. Eur. Respir. J. 47, 938-946 (2016).

28. Chun, F. K., Briganti, A., Shariat, S. F., Graefen, M., Montorsi, F., Erbersdobler, A. et al. Significant upgrading affects a third of men diagnosed with prostate cancer: predictive nomogram and internal validation. BJU Int. 98, 329-334 (2006).

29. Kuroiwa, K., Shiraishi, T., Ogawa, O., Usami, M., Hirao, Y., Naito, S. et al. Discrepancy between local and central pathological review of radical prostatectomy specimens. J. Urol. 183, 952-957 (2010).

30. Bryne, M., Koppang, H. S., Lilleng, R., Stene, T., Bang, G. \& Dabelsteen, E. New malignancy grading is a better prognostic indicator than Broders' grading in oral squamous cell carcinomas. J. Oral. Pathol. Med. 18, 432-437 (1989).

31. Almangush, A., Bello, I. O., Keski-Santti, H., Makinen, L. K., Kauppila, J. H., Pukkila, $M$. et al. Depth of invasion, tumour budding, and worst pattern of invasion: prognostic indicators in early-stage oral tongue cancer. Head Neck. 36, 811-818 (2014).

32. Jesinghaus, M., Strehl, J., Boxberg, M., Bruhl, F., Wenzel, A., Konukiewitz, B. et al. Introducing a novel highly prognostic grading scheme based on tumour budding and cell nest size for squamous cell carcinoma of the uterine cervix. J. Pathol. Clin. Res. 4, 93-102 (2018).

33. Kurokawa, H., Zhang, M., Matsumoto, S., Yamashita, Y., Tomoyose, T., Tanaka, T. et al. The high prognostic value of the histologic grade at the deep invasive front of tongue squamous cell carcinoma. J. Oral. Pathol. Med. 34, 329-333 (2005).

34. Lindenblatt Rde, C., Martinez, G. L., Silva, L. E., Faria, P. S., Camisasca, D. R. \& Lourenco Sde, Q. Oral squamous cell carcinoma grading systems-analysis of the best survival predictor. J. Oral. Pathol. Med. 41, 34-39 (2012).

35. Brandwein-Gensler, M., Smith, R. V., Wang, B., Penner, C., Theilken, A., Broughel, D. et al. Validation of the histologic risk model in a new cohort of patients with head and neck squamous cell carcinoma. Am. J. Surg. Pathol. 34, 676-688 (2010).

36. Li, Y., Bai, S., Carroll, W., Dayan, D., Dort, J. C., Heller, K. et al. Validation of the risk model: high-risk classification and tumour pattern of invasion predict outcome for patients with low-stage oral cavity squamous cell carcinoma. Head Neck Pathol. 7, 211-223 (2013).

37. Rodrigues, P. C., Miguel, M. C., Bagordakis, E., Fonseca, F. P., de Aquino, S. N., Santos-Silva, A. R. et al. Clinicopathological prognostic factors of oral tongue squamous cell carcinoma: a retrospective study of 202 cases. Int. J. Oral. Maxillofac. Surg. 43, 795-801 (2014).

38. Takes, R. P., Rinaldo, A., Silver, C. E., Piccirillo, J. F., Haigentz, M. Jr., Suarez, C. et al. Future of the TNM classification and staging system in head and neck cancer. Head Neck 32, 1693-1711 (2010). 
39. Aydil, U., Duvvuri, U., Kizil, Y. \& Koybasioglu, A. Tumour-node-metastasis staging of human papillomavirus negative upper aerodigestive tract cancers: a critical appraisal. J. Laryngol. Otol. 129, 1148-1155 (2015).

40. Hubert Low, T. H., Gao, K., Elliott, M. \& Clark, J. R. Tumour classification for early oral cancer: re-evaluate the current TNM classification. Head Neck 37, 223-228 (2015).

41. Almangush, A., Pirinen, M., Heikkinen, I., Makitie, A. A., Salo, T. \& Leivo, I. Tumour budding in oral squamous cell carcinoma: a meta-analysis. Br. J. Cancer 118, 577-586 (2018).

42. Zhu, Y., Liu, H., Xie, N., Liu, X., Huang, H., Wang, C. et al. Impact of tumour budding in head and neck squamous cell carcinoma: a meta-analysis. Head Neck 41, 542-550 (2019).

43. Ueno, H., Murphy, J., Jass, J. R., Mochizuki, H. \& Talbot, I. C. Tumour 'budding' as an index to estimate the potential of aggressiveness in rectal cancer. Histopathology 40, 127-132 (2002).

44. Karamitopoulou, E., Zlobec, I., Kolzer, V., Kondi-Pafiti, A., Patsouris, E. S., Gennatas, K. et al. Proposal for a 10-high-power-fields scoring method for the assessment of tumour budding in colorectal cancer. Mod. Pathol. 26, 295-301 (2013).

45. Almangush, A., Youssef, O., Pirinen, M., Sundstrom, J., Leivo, I. \& Makitie, A. A. Does evaluation of tumour budding in diagnostic biopsies have a clinical relevance? A systematic review. Histopathology 74, 536-544 (2019).
46. Grigore, A. D., Jolly, M. K., Jia, D., Farach-Carson, M. C. \& Levine, H. Tumour budding: the name is EMT. Partial EMT. J. Clin. Med. 5, 5 (2016).

47. Makitie, A. A., Almangush, A., Rodrigo, J. P., Ferlito, A. \& Leivo, I. Hallmarks of cancer: tumour budding as a sign of invasion and metastasis in head and neck cancer. Head Neck 41, 3712-3718 (2019).

48. Qiao, Y., Wang, Y., Kang, P., Li, R., Liu, Y. \& He, W. The assessment of the accuracy of clinical preoperative lymph node. Medicine 98, e13778 (2019).

49. De Waal, P. J., Fagan, J. J. \& Isaacs, S. Pre- and intra-operative staging of the neck in a developing world practice. J. Laryngol. Otol. 117, 976-978 (2003).

50. Keski-Santti, H., Atula, T., Tornwall, J., Koivunen, P. \& Makitie, A. Elective neck treatment versus observation in patients with $\mathrm{T} 1 / \mathrm{T} 2 \mathrm{~N} 0$ squamous cell carcinoma of oral tongue. Oral Oncol. 42, 96-101 (2006).

51. D'Cruz, A. K. \& Dandekar, M. R. Elective versus therapeutic neck dissection in the clinically node negative neck in early oral cavity cancers: do we have the answer yet? Oral Oncol. 47, 780-782 (2011).

52. Lowe, V. J., Duan, F., Subramaniam, R. M., Sicks, J. D., Romanoff, J., Bartel, T. et al. Multicenter trial of [18 fffluorodeoxyglucose positron emission tomography/ computed tomography staging of head and neck cancer and negative predictive value and surgical impact in the No neck: results from ACRIN 6685. J. Clin. Oncol. 37, 1704-1712 (2019). 